\title{
Deterministic Model for Acute Myelogenous Leukemia Classification
}

\author{
Monica Madhukar(mayaa.madhukar@gmail.com), Sos Agaian(sos.agaian@utsa.edu), \\ Anthony T.Chronopoulos(atc@cs.utsa.edu) \\ Department of Electrical and Computer Engineering \\ University Of Texas at San Antonio \\ One UTSA Circle, San Antonio, Texas 78249
}

\begin{abstract}
Leukemia is a type of cancer that affects the blood and the bone marrow. Manual data analysis is time consuming and not accurate. Attempts to build partial/full automated systems based on segmentation and classification of cells are present in literature, but they are still in prototype stage. Most of the existing automatic systems extract features of the sub-images instead of the complete blood smear. [29]. The main objective of this paper is to a) demonstrate that the classification of peripheral blood smear images containing multiple nuclei can be fully automated, b) to validate the segmented images using holdout cross validation method. The method has been evaluated using a set of 50 images (with 25 abnormal samples and 25 normal samples) obtained from American Society of Hematology [22]. The computer simulations show that the proposed system robustly segments and classifies Acute Myelogenous Leukemia based on complete microscopic blood images. $93.5 \%$ of the cases were correctly classified by the program, suggesting that the method yields good results in terms of classification of leukemia. The developed system can be used as ancillary/backup service to the physician
\end{abstract}

Keywords- Classification; Segmentation; Acute Myelogenous leukemia; Feature Extraction

\section{INTRODUCTION}

White blood cells (WBC) or leukocytes play a significant role in the diagnosis of different diseases (including Leukemia), and therefore, extracting information about that is valuable for hematologists. Leukemia refers to a progressive, malignant disease of the blood-forming organs. Complete blood count process is the first step for leukemia screening [4]. The pathology is characterized by the uncontrolled accumulation of immature white blood cells. The four main types of leukemia are Acute Myelogenous Leukemia (AML); Acute Lymphoblastic Leukemia (ALL); Chronic Myeloid Leukemia (CML); Chronic Lymphocytic Leukemia (CLL). In this paper, we build up a decision support tool to improve classification of Acute Myelogenous Leukemia (AML). AML is a fast-growing cancer of the blood and bone marrow. In AML, the bone marrow produces many unformed cells called blasts. Blasts usually develop into white blood cells that are used for defense mechanism in the body. However, the blasts are not fully formed in AML and hence cannot fight infections. Acute Myelogenous leukemia is often difficult to diagnose since the precise cause of AML is still unknown. Also the symptoms of the disease are very similar to flu or other common diseases, like fever, weakness, tiredness, or aches in bones or joints. Since there is no staging for acute myelogenous leukemia, choosing the type of treatment can vary from chemotherapy, radiation therapy, bone marrow transplant, and biological therapy. [1]. AML is one of the most common types of leukemia in adults, affecting more than 10,000 adults that are diagnosed every year in the U.S. AML also makes up 15 to 20 percent of childhood leukemia. About 500 children and adolescents in the U.S. each year are affected by AML. [2]

Despite of advanced techniques such as flow cytometer, immunophenotyping, molecular probing etc, microscopic examination of blood slides still remains as the standard leukemia diagnosis technique. This analysis suffers from time delays and it presents not a standardized accuracy since it depends on operator's capabilities and tiredness. So there is always a need for a cost effective and robust automated system for leukemia screening which can greatly influence the decision without being affected by the operator's fatigue.

In the proposed system, the microscopic blood images containing multiple nuclei are exposed to series of preprocessing steps which include color correlation, and contrast enhancement. By performing $\mathrm{K}$-means clustering on the resultant images, the nuclei of the cells invested with cancer are obtained. Currently five main features are used by automated systems for early detection: cell size, color, shape, density and granularity. In literature different methods have been employed for leukemia classification. For instance, [29] employs neural classifier to distinguish subtypes of leukocytes; a fuzzy based two stage color segmentation strategy was employed in [30]; [8] uses cell segmentation based on active contour models; Color segmentation procedure applied to leukocyte images is described in [9]; [31] incorporated Expectation-Maximization algorithm for blood cell segmentation; [10] makes use of shape analysis into WBC segmentation, by considering features such as cell size, granularity, density and shape; However, the identification task is usually difficult due to the variety of features and the often unclear images which cause missing out on vital indicators as to which form of leukemia is being observed. Meeting real clinical demands becomes all the more challenging due to complex nature of the blood smear images and variation in slide preparation. Also, most of the existing systems extract features of the sub-images instead of the complete blood smear. 
We had earlier developed a system that classified acute lymphoblastic leukemia based on shape features and color features [32]. In this paper we employ our techniques to see how effectively our system can classify Acute Myelogenous Leukemia based on texture features.

The main goal of this paper is to demonstrate that the classification of peripheral blood smear images containing multiple nuclei can be fully automated.

The paper is structured as follows; Section 2 focuses in detail on the proposed system overview and compares it with t12he literature. Section 3 and Section 4 deal with color correlation and segmentation of the images. Section 5 summarizes the extraction of features of the segmented images, which then are subsequently classified in Section 6. Section 7 provides the performance evaluation of the system based on the parameters extracted.

\section{AML SYSTEM OVERVIEW}

In this section we briefly discuss the structure of the system. Many of the image-processing systems for leukemia detection in the literature are still at prototype stage [5-7]. Some systems have been proposed as techniques to refine the segmentation (i.e., to solve a particular cluster of cells such as in [11] or to improve membrane segmentation as in [12] or to detect incorrect segmentations of white cells as in [13]. Also most of systems developed, work on sub-images where only one nucleus per image is presented under the field of view and hence the features are being extracted only for sub-images instead of whole images. Our goal is to overcome this and also increase the accuracy of the classifier system.

The proposed approach aims to present a more robust system with an efficient segmentation of blood images for high performance. To achieve this goal, the system we propose follows four main processing steps (See Figure 1 for complete system overview):

1) To preprocess the image in order to reduce background non-uniformities and perform color correlation;

2) To employ segmentation on whole images by combining different methods in order to exploit all the available a-priori information and thereby achieving a robust identification of the nuclei of the white cells ;

3) To extract different sets of features for a database of images.

4) To run the classifier system and validate the output based on the results obtained.

\section{PRE-PROCESSING: COLOR-CORRELATION}

An irregular background illumination is one the common drawbacks of a camera-acquired image of a blood sample. Noise may be accumulated during image acquisition and due to excessive staining. The background non-uniformities are smoother than the cells present in the image. Hence it is assumed that background has lower spatial frequencies than the cells. Thus selective filtering can be applied to the test images to suppress the background non-uniformities.

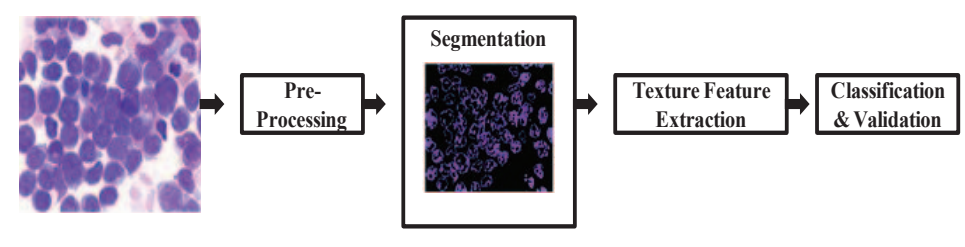

Figure 1: System Over-view

Typically images generated by digital microscopes are usually in RGB color space which is difficult to segment. In practice the blood cells and image background varies strongly with respect to color and intensity. This results due to multiple reasons such as by camera settings, varying illumination and aging stain. In order to make the cell segmentation robust with respect to these variations an adaptive procedure is used: the $\mathrm{RGB}$ input image is converted into the CIELAB color space.

The L*a*b* color space is a color representation technique which is basically used to reduce the color dimension from three to two in comparison to RGB. The $\mathrm{L}^{*} \mathrm{a} * \mathrm{~b}^{*}$ space consists of a luminosity layer $\mathrm{L}^{*}$, chromaticity layer a* and chromaticity layer $b^{*}$. Here the color information is represented in two components i.e. $a^{*}$ and $b^{*}$. Due to less color dimension $\mathrm{L}^{*} \mathrm{a} \mathrm{b}^{*}$ color space is mostly employed in color based clustering. In a uniform color scale, the differences between points plotted in the color space correspond to visual differences between the colors plotted. The CIELAB is organized in cube form [14].

Also $\mathrm{L} * \mathrm{a} * \mathrm{~b}$ color is designed to approximate human vision and it aspires perceptual uniformity, and its L component closely matches human perception of lightness. In the present work microscopic images are converted from RGB color space to $\mathrm{L} * \mathrm{a} * \mathrm{~b} *$ before clustering. Figure 2 shows the results of L*a*b space conversion.

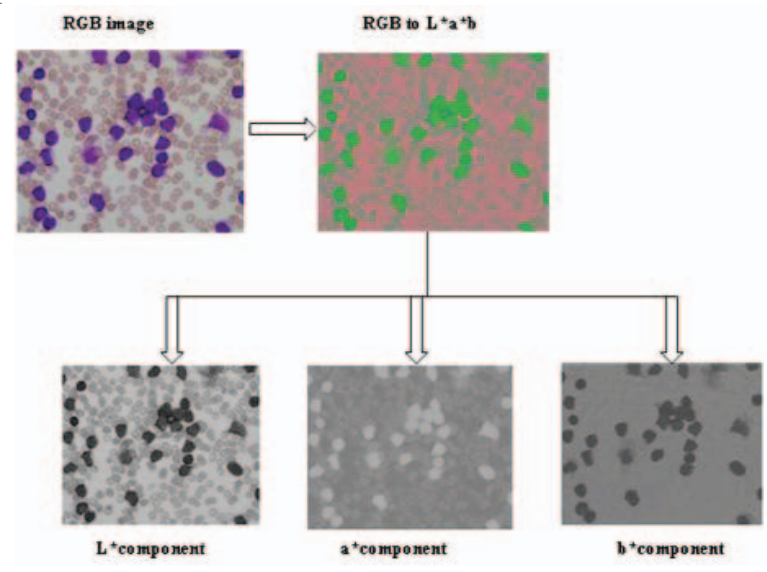

Figure 2: L*a*b Color Correlation Results 


\section{SEGMENTATON: K-MEANS CLUSTERING}

This section discusses the segmentation technique used to extract the nuclei from the complete blood smear images. Segmentation plays a key role since the efficiency of subsequent feature extraction and classification relies greatly on the correct segmentation of the blasts. Many algorithms for segmentation have been developed for gray level images. Cell segmentation using active contour models is presented in [12]; Color segmentation procedure applied to leukocyte images are described in [9]; a two step segmentation process using HSV color model is used in [16].

Segmentation is performed for extracting the nuclei of the leukocytes using color based clustering. A k-means clustering procedure is used to assign every pixel to one of the clusters. Each pixel of an object is classified into $k$ clusters based on the corresponding $* \mathrm{a}$ and $* \mathrm{~b}$ values in $\mathrm{L} * \mathrm{a} * \mathrm{~b}$ color space. These clusters correspond to nucleus (high saturation), background (high luminance, low saturation), and other cells (e. g., erythrocytes and leukocyte cytoplasm). Every pixel is assigned to one of these classes using the properties of the cluster center. Now each pixel in the L*a*b color space is classified into any of the $k$ clusters by calculating the Euclidean distance between the pixel and each color indicator. Each pixel of the entire image will be labeled to a particular color depending on the minimum distance from each indicator. We consider only the cluster which contains the blue nucleus, which is required for the feature extraction.

K-means is one of the simplest unsupervised learning algorithms that solve the well known clustering problem. It is composed of following steps:

1. Place K points (initial group centroids) into the space represented by the objects that are being clustered.

2. Assign each object to the group that has the closest centroid.

3. Recalculate the positions of the K centroids, when all objects have been assigned,

4. Repeat Steps 2 and 3 until the centroids no longer move. This produces a separation of the objects into groups from which the metric to be minimized can be calculated.

Attempts were made mostly to classify only sub-images of acute myelogenous leukemia. We attempt to overcome this drawback by segmenting the complete image comprising of multiple myeloblasts. We consider microscopic blood images of size $184 \times 138$. The input images after undergoing $\mathrm{L}^{*} \mathrm{a} * \mathrm{~b}$ color conversion are subjected to K-means clustering. The cluster considered contains only the blue nuclei. Figure 3 shows segmented output of the cell nucleus image after applying K-means algorithm.

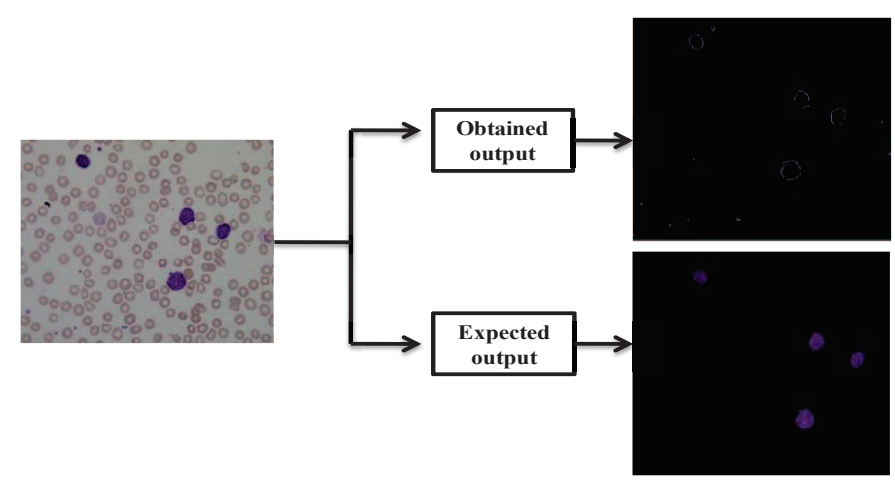

Figure3: Examples of Segmentation using K-means Clustering

While performing K-means segmentation, it was observed that in some of the segmented images, we obtained only the nuclei edges (refer Figure 3(b)) as opposed to the whole nuclei. Hence to make the system more robust, all segmented images were made to undergo morphological filtering [15]. Texture based features were then extracted from the resultant images.

\section{FEATURE EXTRACTION}

This section discusses about the future vectors extracted from the segmented images. Feature extraction in image processing is a technique of redefining a large set of redundant data into a set of features of reduced dimension. Transforming the input data into the set of features is called feature extraction. Feature extraction plays a crucial role in obtaining relevant information from the input data. Our image database was classified based on 23 different future vectors which include Hausdorff dimension and the well-known Haralick features.

\section{A. GLCM Features}

Our paper proposes efficient classification of cancer cells based on famous Haralick features [17]. These texture features based on Grey Level Co-occurrence Matrix (GLCM) is one of the most widely used techniques for texture analysis. For a given image composed of pixels with a specific gray level or intensity, GLCM offers a tabulation of how frequently different combinations of gray levels co-occur in an image section [18].In other words it is an estimate of image properties related to second order statistics introduced by Haralick [17].

The proposed classification is based on the values of the different Haralick features implemented on color segmented images. These were used as a primary component which aids in distinguishing cancerous cells from non-cancerous cells. This section summarizes briefly some of the main Haralick's texture features used.

Identify applicable sponsor/s here. (sponsors) 
Framework for the GLCM [19]:

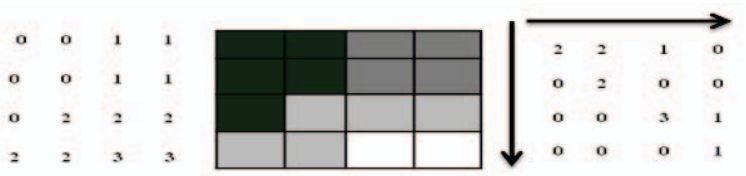

Figure 4: The computed GLCM P(1,0) from a four grey level image.

GLCM texture considers the relation between two pixels at a time: one being the reference pixel and other being the neighboring pixel. In Figure4, the neighboring pixel is chosen to be the one to the right of each reference pixel. Each pixel within the window becomes the reference pixel in turn, starting in the upper left corner and proceeding to the lower right. Pixels along the right edge have no right hand neighbor, so they are not used for this count.

Haralick defined several coefficients, which can be calculated from the normalized GLCM, $\mathrm{P}_{\mathrm{i}, \mathrm{j}}$ (where $i$ refers to the row number and $j$ refers to the column number) [17]. Some of the main Haralick's coefficients on GLCM for texture analysis considered in our study are:

- Contrast: The contrast feature is a difference moment of the regional co-occurrence matrix and is a measure of the contrast or the amount of local variations present in an image. It is also called the sum of squares variance.

$$
\sum_{i, j=0}^{N-1} P_{i, j}(i-j)^{2}
$$

- Homogeneity: Homogeneity weighs values by the inverse of the contrast weight, with weights decreasing exponentially away from the diagonal.

$$
\sum_{i, j=0}^{N-1} \frac{P_{i, j}}{1+(i-j)^{2}}
$$

- Energy: Also known as uniformity or Angular Second Moment (ASM), it is a measure of homogeneity of image or in other words represents orderliness.

$$
\sum_{i, j=0}^{N-1} P_{i, j}^{2}
$$

- Entropy: This parameter measures the disorder of an image. When the image is not texturally uniform, entropy is very large. It is usually classified as a first degree measure.

$$
\sum_{i, j=0}^{N-1} P_{i, j}\left(-\ln P_{i, j}\right)
$$

- Correlation: The correlation texture measures the linear dependency of grey levels on those neighboring pixels.

$$
\sum_{i, j=0}^{N-1} P_{i, j} \frac{\left(i-\mu_{i}\right)\left(j-\mu_{i}\right)}{\sqrt{\sigma_{i}^{2} \sigma_{j}^{2}}}
$$

\section{B. Fractal Dimension}

The fractal dimension, $D$, is a statistical quantity that gives an indication of how completely a fractal appears to fill space. There are many specific methods to calculate fractal dimension and box-counting mechanism is the simplest to implement. The algorithm offers two main advantages over other methods: one, it can be applied to any type of image irrespective of the complexity and two, it is very easy to implement in case of using a computer. The following approximation depicts 'box-counting' fractal dimension derived from Hausdorff coverage dimension.

$$
H D=\frac{\log (R)}{\log (R(s))}
$$

Where, $R$ is the number of squares in the superimposed grid and $\mathrm{R}(s)$ is the number of occupied squares or boxes (box count). Higher HD implies higher degree of roughness.

In a box counting algorithm the number of boxes covering the point set is a power law function of the box size. Fractal dimension is estimated as the exponent of such power law. Myeloblasts can be identified using their perimeter roughness of their nuclei. Hausdorff dimension is considered as an essential feature our proposed system.

In a synthetic representation, the algorithm for determining the"box counting" dimension is the following [20 ][21]:

1. Binary image in obtained from the gray-level image of the blood sample;

2. The region to be analyzed (here the nuclei) is selected;

3. The box-counting dimension is calculated by counting the number of cubes $N(s)$ that overlap with at least a point from the region of interest.

4. Logarithm is applied to the obtained values which then are graphically represented by a curve with a slope.

The Hausdorff Dimension turned out to be a crucial feature in our system, particularly since we considered whole images of the blood sample. In whole images, the number of nuclei under the field of view was much higher for a cancerous case as opposed to the non-cancerous case. This resulted in steep difference in box-count between the two cases and thereby proved to be an effective feature. This is illustrated in the Figure 5.
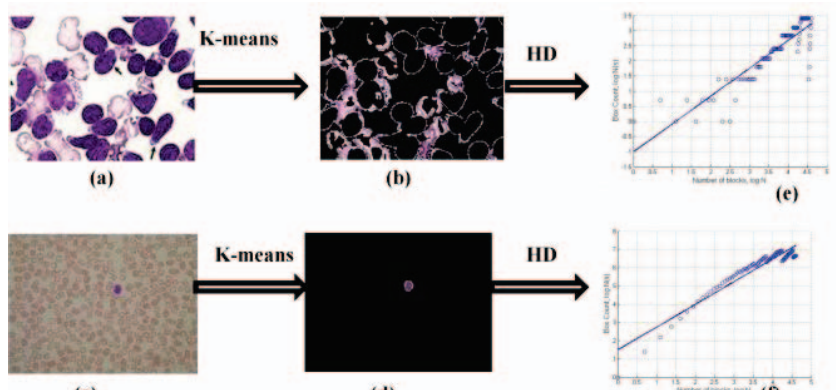

(d)

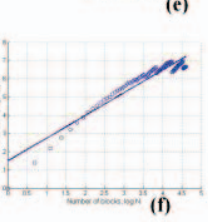

Figure5: Fractal Dimension Results 


\section{COMPUTER SIMULATION [26]}

The method has been evaluated using a set of 50 images obtained from American Society of Hematology [22]. All images are in JPG format with 24 bit color depth has a size of $184 \times 138$ pixels. The features are extracted from the segmented images and classified using the Support Vector Machine (SVM). A Support Vector Machine (SVM) is a classifier which constructs an $\mathrm{N}$-dimensional hyper plane that optimally separates the data into two sets. In SVM literature, a predictor variable is called an attribute, and a transformed attribute that is used to define the hyper plane is called a feature. Feature Selection is the method of choosing the most apt representation. A set of features that describes one case (i.e., a row of predictor values) is called a vector. Hence SVM aims at finding the optimal hyper plane that separates clusters of vector based on their target variables. The vectors near the hyper plane are the support vectors. Classification of images is followed by validation.

Cross-Validation is essentially performed to compare the performance of two or more different algorithms and choose the one that is the best for the given set of data. A statistical method of evaluating and comparing learning algorithms by dividing data into two sets(one set used to learn or train a model and the other used to validate the model). The crossvalidation type employed in our system is hold-out cross validation. In hold-out validation, an independent test set is used, to avoid over-fitting. This can be achieved by splitting the available data into two non-overlapped parts: one for testing and the other for training. The test data is held out and not considered during training.

\section{PERFORMANCE EVALUATION}

In order to ensure the effectiveness of our system, we employed certain measures based on which decisions were made.

Precision, Specificity, Sensitivity and F-Measure are all defined in relation to the possible outcomes of the classifier system. When attempting to classify a specimen, there are four possible outcomes [28]: True Positive (cancerous cell correctly identified), False Positive (non-cancerous cells identified as cancerous), True Negatives (non-cancerous correctly identified), False Negatives (Cancerous cells identified as non-cancerous).

\begin{tabular}{|l|c|}
\hline \multicolumn{1}{|c|}{ Parameters } & Formulae \\
\hline $\begin{array}{l}\text { Sensitivity -also called recall denotes the test's } \\
\text { ability to identify positive results }\end{array}$ & $\mathrm{TP} /(\mathrm{TP}+\mathrm{FN})$ \\
\hline $\begin{array}{l}\text { Specificity denotes the test's ability to identify } \\
\text { negative results. }\end{array}$ & $\mathrm{TN} /(\mathrm{TN}+\mathrm{FP})$ \\
\hline $\begin{array}{l}\text { Precision -gives the proportion of subjects with } \\
\text { positive results who are correctly identified. }\end{array}$ & $\mathrm{TP} /(\mathrm{TP}+\mathrm{FP})$ \\
\hline $\begin{array}{l}\text { F-Measure- is a metric that gives the harmonic mean } \\
\text { of Precision and Sensitivity. It is the overall } \\
\text { classification performance }\end{array}$ & $\frac{2 \times \text { Precision } \mathrm{x} \text { Sensitivy }}{\text { Precision }+ \text { Sensitivity }}$ \\
\hline
\end{tabular}

Table 1: Performance Evaluation parameters
The following table summarizes the value obtained for the above measures.

\begin{tabular}{|l|l|}
\hline Measure & Value \\
\hline Specificity & $91 \%$ \\
\hline Sensitivity & $83 \%$ \\
\hline Precision & $90 \%$ \\
\hline F-Measure & $88 \%$ \\
\hline
\end{tabular}

Table 2: Performance Evaluation Statistics

VIII. CONCLUSION

Thus this article aims at building a robust classifier system which segments and classifies microscopic blood images. The main advantage of this proposed scheme over existing schemes is that, the developed system effectively classifies the complete blood smear images of Acute Myelogenous Leukemia (AML) containing multiple nuclei, while existing systems mostly consider only those images which have one cell under the field of view. The discussed set of texture features were extracted for 50 complete blood smear images of AML. The SVM classifier $93.5 \%$ of the AML blood smear images correctly. Future work suggests that, to present the output interactively, a Graphical User Interface (GUI) could be developed. Also, a combined system could be developed to classify all the types of Acute Leukemia.

\section{ACKNOWLEDGEMENT}

This research has been partially supported by NSF Grant (HRD-0932339) to the University of Texas San Antonio. We are also thankful to American Society of Hematology for providing us with high quality image database.

\section{REFERENCES}

[1] Acute Myelogenous Leukemia, Acute Lymphocytic Leukemia. [Online]. Available:http://www.acute-myelogenousleukemia.com/html/complications.html.

[2] Acute Myelogenous Leukemia (AML) in Children , [Online]. Available:http://www.chop.edu/service/oncology/cancersexplained/acute-myeloid-leukemia-in-children.html

[3] J.Poomcokrak and C.Neatpisarnvanit,"Red Blood Cells Extraction and Counting", [Online]. Available: http://www.kmitl.ac.th/ijabme/proceedings/bmeicon08/pdf/Session4/110

[4] G. P. M. Priyankara, O. W Seneviratne, R. K. O. H Silva, W. V. D Soysa and C. R. D. Silva, "An Extensible Computer Vision Application for Blood Cell Recognition and Analysis", 2006.

[5] Serbouti, S.;Duhamel, A.; Harms, H.; Gunzer, U.; Aus, U.M.; Mary, J.Y.;Beuscart, R.; "Image segmentation and classification methods to detect leukemias," in Proc. International conference of IEEE engineering in Medicine and Biology society, 1991. Pages 260261.(1991)

[6] Foran, D.J.; Comaniciu, D.;Meer, P.; Goodell, L.A.; Centre for Biomed. Imaging \& Inf, UMDNJ, Piscataway, NJ, USA, "Computer-assisted discrimination among malignant lymphomas and leukemia using immunophenotyping, intelligent image repositories, and telemicroscopy.," IEEE Transactions on Information Technology in Biomedicine, vol. 4, no. 4, pp. 265-273, (2000). 
[7] K.S. Kim, P.K. Kim, J.J. Song, and Y.C. Park, "Analyzing blood cell image do distinguish its abnormalities," in Proc. ACM International Conference on Multimedia, (2002).

[8] Qingmin Liao; Yingying Deng; Dept. of Electron. Eng., Tsinghua Univ., Beijing, China "An accurate segmentation method for white blood cell images". in Proc. IEEE Int. Symp. on Biomedical Imaging, (2002).

[9] J. S. Suri, S. K. Setarehdan, and S. Singh, "Advanced algorithmic approaches to medical image segmentation: state-of-the-art application in cardiology, neurology, mammography and pathology", $541-558$. Springer, (2001).

[10] Guclu Ongun, Ugur Halici, Leblebicioglu, K.; Atalay, V.; Beksac, M.; Beksac, S.; Dept. of Electr. \& Electron.Eng., Middle East Tech.Univ., Ankara, "Feature Extraction and Classification of Blood Cells for an Automated Differential Blood Count System" in Neural Networks, 2001. Proceedings. IJCNN '01. Pages 2461-2466 vol.4.,(2001)

[11] Nilsson, B.; Heyden, A., "Model-based segmentation of leukocytes clusters," in Proc. of International Conf. on Pattern Recognition, pp. 727-730, vol.1,(2002).

[12] Guclu Ongun, Ugur Halici, Leblebicioglu, K.; Atalay, V.; Beksac, M.; Beksac, S.; Dept. of Electr. \& Electron.Eng., Middle East Tech.Univ., Ankara, "Feature Extraction and Classification of Blood Cells for an Automated Differential Blood Count System" in Neural Networks, 2001. Proceedings. IJCNN '01. Pages 2461-2466 vol.4.,(2001)

[13] P. Bamford and B. Lovell, "Method for accurate unsupervised cell nucleus segmentation," in Proc. of the Engineering in Medicine and Biology Society Conference, pages 2704-2708, vol.3,(2001).

[14] CIE L*a*b Color Scale - Hunter Lab, [Online].Available: http://www.hunterlab.com/appnotes/an07 96a.pdf.

[15] Scotti,F., “ Automatic Morphological Analysis for Acute Leukemia Identification in Peripheral Blood Microscope Images",in 2005 IEEE International Conference on Computational Intelligence for Measurement Systems and Application. CISMA. Pages 96-101,(2005).

[16] Sinha, N.; Ramakrishnan, A.G.; Dept. of Electr. Eng., Indian Inst. of Sci., Bangalore, India, "Automation of differential blood count". In Proceedings Conference on Convergent Technologies for Asia-Pacific Region, volume 2, pages 547-551, (2003).

[17] R. M. Haralick, K. Shanmugam and I. Dinstein, "Textural features for image classification", IEEE Trans. Syst. Man Cybern, Vol. 3, 1973, pp. 610-621.

[18] About the GLCM and textures. [Online]. Available: http://support.echoview.com/WebHelp/erence/Algorithms/Operators/GL CM textures/About the GLCM and textures.htm
[19] The GLCM Texture Tutorial by Mryka Hall-Beyer, [Online]. Available: http://www.fp.ucalgarby.ca/mhallbey/

[20] A. P. Pentland, "Fractal based description of natural scenes ." IEEE Transactions on Pattern Analysis and Machine Intelligence, 6:661 - 674, (1984).

[21] Sztojanov, I.; Voinea, V.; Stanica, J.-L.; Popescu-Mina, C.; Univ. Politeh. of Bucharest, Bucharest, Romania," Fractal technologies for image processing in biology", in 3rd International Workshop on Soft Computing Applications, 2009. SOFA '09. Pages 139-144,(2009).

[22] American Society Of Hematology - Helping hematologists conquer blood diseases worldwide. [Online]. Available: http://www.hematology.org/About-ASH/

[23] C.J.C. Burges, "A Tutorial on Support Vector Machines for Pattern Recognition", Data Mining and Knowledge Discovery, vol.2, No.2, pp.121-167,(1998).

[24] B. Scholkopf, A. J. Smola, "Learning with Kernels: Support Vector Machines, Regularization Optimization and Beyond", The MIT Press, (2002).

[25] N. Cristianini, J. Shawe-Taylor, "An Introduction to Support Vector Machines and other kernel-based learning Methods", Cambridge University Press 2000.

[26] Benjamin M. Rodriguez, Gilbert L. Petersona, Sos S. Agaian, "Steganography Anomaly Detection Using Simple One-Class Classification", Proceedings of SPIE (2007).

[27] Payam aeilzadeh, Lei Tang, Huan Liu, Arizona State University, "Cross-Validation", in Encyclopedia of Database Systems EDBS), Editors: Ling Liu and M. Tamer Özsu. Springer, pp6. (2009).

[28] Sensitivity and Specificity.[Online]. Available : http://en.wikipedia.org/wiki/Sensitivity and specificity

[29] Piuri,V.; Scotti,F.; "Morphological Classification of Blood leucocytes by microscope images", 2004 IEEE International Conference on Computational Intelligence for Measurement Systems and Applications (CIMSA), doi: 07 March 2005, Pages: 103-108.

[30] Mohapatra, S.; Samanta, S.S.; Patra, D.; Satpathi, S.; IPCV Lab., Nat. Inst. of Technol., Rourkela, India, "Fuzzy Based Blood Image Segmentation for Automated Leukemia Detection", in Devices and Communications (ICDeCom), Pages 1-5. (2011).

[31] Neelam Sinha, A.G.Ramakrishnan, 'Blood Cell Segmentation Using EM Algorithm".

[32] Monica Madhukar, Sos Agaian, Anthony.T.Chronopoulos, “ New Decision Support Tool for Acute Lymphoblastic Leukemia Classification", Image Processing: Algorithms and Systems X; Proc. SPIE 8295,(2012). 